\title{
COHOMOLOGICAL ASPECTS OF HYPERGRAPHS
}

\author{
F. R. K. CHUNG AND R. L. GRAHAM
}

\begin{abstract}
By a $k$-graph we will mean a collection of $k$-element subsets of some fixed set $V$. A $k$-graph can be regarded as a $(k-1)$-chain on $2^{V}$, the simplicial complex of all subsets of $V$, over the coefficient group $\mathbb{Z} / 2$, the additive group of integers modulo 2 . The induced group structure on the $(k-1)$ chains leads to natural definitions of the coboundary $\delta$ of a chain, the cochain complex of $C=\left\{C^{k}, \delta\right\}$ and the usual cohomology groups $H^{k}(C ; \mathbb{Z} / 2)$. In particular, it is possible to construct what could be called "higher-order" coboundary operators $\delta^{(i)}$, where $\delta^{(i)}$ increases dimension by $i$ (rather than just 1).

In this paper we will develop various properties of these $\delta^{(i)}$, and in particular, compute the corresponding c.shomology groups for $2^{V}$ over $\mathbb{Z} / 2$. It turns out that these groups depend in a rather subtle way on the arithmetic properties of $i$.
\end{abstract}

\section{INTRODUCTION}

Among the most fundamental objects occurring in combinatorics are the socalled $k$-uniform hypergraphs, or $k$-graphs, for short. A $k$-graph is simply a collection of (distinct) $k$-element subsets, called edges, of some fixed set $V$. Because of the great generality of this definition, virtually any problem in combinatorics can be phrased in terms of a corresponding question about an appropriate class of $k$-graphs. For example, much of the field of Ramsey theory (cf. [GRS90]) can be interpreted simply as the study of chromatic numbers of certain $k$-graphs (where the chromatic number of a $k$-graph is the minimum number of classes into which $V$ can be partitioned so that no edge is contained entirely in one class). For a full discussion of $k$-graphs, the reader should consult Berge [B89].

From a somewhat different point of view, $k$-graphs can also be regarded as $(k-1)$-chains on $2^{V}$, the simplicial complex of all subsets of $V$, over the coefficient group $\mathbb{Z} / 2$, the additive group of integers modulo 2 (so that the orientation of simplices is irrelevant). From this perspective, the induced group structure on the $(k-1)$-chains leads to natural definitions of the coboundary $\delta$ of a chain, the cochain complex $C=\left\{C^{k}, \delta\right\}$ and the usual cohomology groups $H^{k}(C ; \mathbb{Z} / 2)$. (For an excellent discussion of these concepts, the reader is referred to Munkres [M84].)

Received by the editors September 27, 1990.

1991 Mathematics Subject Classification. Primary 05C65, 05E99, 55N20.

(c) 1992 American Mathematical Society $0002-9947 / 92 \$ 1.00+\$ .25$ per page 
In particular, it is possible to construct a class of what could be called "higherorder" coboundary operators $\delta^{(i)}$, where $\delta^{(i)}$ increases dimension by $i$ (rather than just 1). Thus, if $G$ is a $k$-graph then $\delta^{(i)} G$ will be a $(k+i)$-graph. These higher order $\delta^{(i)}$ were in fact introduced by S. T. Hu [H49, H50, H52] in 1949, who showed that they satisfy all but one of the Eilenberg-Steenrod axioms for a cohomology theory.

It turns out that in recent work of the authors and R. M. Wilson [CGW89, CG90, CG91] investigating aspects of random-like behavior in $k$-graphs, these higher-order coboundary operators arose in a natural way, and played an important role in settling several fundamental conjectures there.

In this paper we will develop further properties of these $\delta^{(i)}$, and in particular, compute the corresponding cohomology groups for $2^{V}$ over $\mathbb{Z} / 2$. As will be seen (Theorem 4), these groups depend in a rather subtle way on the arithmetic properties of $i$, and in particular, on the representation of $i$ to the base 2. We point out that there is a considerable body of work dealing with cohomological aspects of 3-uniform hypergraphs (cf. [MS75, C77, C78, S76, ST81, $\mathrm{ML83,} \mathrm{Z81}$, We84, CW86]). In some sense, our results can be considered as the beginnings of a natural extension of this work to general hypergraphs.

\section{DEFINITIONS AND BASIC PROPERTIES}

Let $V$ be a finite set of cardinality $|V|=n$, and let $\left(\begin{array}{l}V \\ k\end{array}\right)$ denote the family of $k$-element subsets of $V$. We denote by $C_{k}=C_{k}(V)$ the vector space over $\mathbb{Z} / 2$ (the integers modulo 2) generated by the $X \in\left(\begin{array}{l}V \\ k\end{array}\right)$. The elements of $C_{k}$ are called $k$-graphs (on $V$ ). Thus, each $k$-graph $G \in C_{k}$ can be written as

$$
G=\sum_{X \in\left(\begin{array}{l}
V \\
k
\end{array}\right)} \chi_{G}(X) X \quad \text { where } \chi_{G}:\left(\begin{array}{l}
V \\
k
\end{array}\right) \rightarrow \mathbb{Z} / 2
$$

We will sometimes write this as $G=\left(V, \chi_{G}\right)$, or $G=G^{(k)}(n)$, if we wish to emphasize that $G$ is a $k$-graph on a set $V$ of $n$ vertices. The elements of $E=E(G):=\chi_{G}^{-1}(1)$ are called the edges of $G$, and we will also occasionally write $G=(V, E)$.

For $k<0$ or $k>n, C_{k}$ consists of the single element 0 , the identity element of $\mathbb{Z} / 2$. With the convention that $\left(\begin{array}{l}V \\ 0\end{array}\right)$ consists of the single element $\varnothing$, the generic element of $C_{0}$ is

$$
G^{(0)}=\sum_{X \in\left(\begin{array}{c}
V \\
0
\end{array}\right)} \chi_{G^{(0)}}(X) X=\chi_{G^{(0)}}(\varnothing) \varnothing .
$$

We define $G_{0}^{(0)}$ to be the 0 -graph having $\chi_{G^{(0)}}(\varnothing)=0$, so that $G_{0}^{(0)}=0 \in C_{0}$. Similarly, we define $G_{1}^{(0)}$ to be the (other) 0 -graph having $\chi_{G^{(0)}}(\varnothing)=1$. Thus, $G_{1}^{(0)}=\varnothing \in C_{0}$. (This convention will be useful later.) The group addition in $C_{k}$ satisfies

$$
\chi_{G+G^{\prime}} \equiv \chi_{G}+\chi_{G^{\prime}} \quad(\bmod 2) \text { for } G, G^{\prime} \in C_{k} .
$$

We will often suppress the dependence of quantities on $k$ when the meaning is clear, e.g., 0 will denote the zero element in $C_{k}$ for every $k$.

For $p \geq 1$, we define the $p$-coboundary operator $\delta^{(p)}: C_{k} \rightarrow C_{k+p}$ as follows: 
For $G=\left(V, \chi_{G}\right) \in C_{k}, \delta^{(p)} F=\left(V, \chi_{F}\right) \in C_{k+p}$ where for $Y \in\left(\begin{array}{c}V \\ k+p\end{array}\right)$,

$$
\chi_{F}(Y):=\sum_{X \in\left(\begin{array}{l}
Y \\
k
\end{array}\right)} \chi_{G}(X) .
$$

(As remarked earlier, $\delta^{(p)}$ should actually be written $\delta_{k}^{(p)}$; we will omit the index $k$ when context makes it clear.) It is easily checked that $\delta^{(p)}$ is a vector space homomorphism, so we have a natural cochain complex $\left(C, \delta^{(p)}\right)$ on $C=$ $\bigcup_{k} C_{k}$ :

$$
\ldots \stackrel{\delta^{(p)}}{\rightarrow} C_{k} \stackrel{\delta^{(p)}}{\rightarrow} C_{k+p} \stackrel{\delta^{(p)}}{\rightarrow} \cdots
$$

(cf. Munkres [M84]).

Actually, (2.2) represents $p$ disjoint cochain complexes, depending on the residue class of $k$ modulo $p$. One of our goals will be to compute the cohomology groups of $\left(C, \delta^{(p)}\right)$ over $\mathbb{Z} / 2$ (see $\left.\S 5\right)$.

Fact 2.1. $\delta^{(p)} \circ \delta^{(p)}=0$ where 0 denotes the map sending everything to the zero element in the corresponding $C_{k}$.

Proof. For $G=\sum_{X \in\left(\begin{array}{c}v \\ k\end{array}\right)} \chi_{G}(X) X \in C_{k}$, we have

$$
\begin{aligned}
\delta^{(p)} \circ \delta^{(p)} G & =\sum_{Z \in\left(\begin{array}{c}
V \\
k+2 p
\end{array}\right)}\left(\sum_{Y \in\left(\begin{array}{c}
Z \\
k+p
\end{array}\right)}\left(\sum_{X \in\left(\begin{array}{l}
Y \\
k
\end{array}\right)} \chi_{G}(X)\right) Z\right) \\
& =\sum_{X \subset Y \subset Z} \chi_{G}(X) Z=\sum_{X \subset Z}\left(\begin{array}{c}
2 p \\
p
\end{array}\right) \chi_{G}(X) Z=0
\end{aligned}
$$

since $\left(\begin{array}{c}2 p \\ p\end{array}\right) \equiv 0(\bmod 2)$ for $p \geq 1$.

More generally, we have the following. For $x \in \mathbb{Z}$, write $x=\sum_{i \geq 0} x(i) 2^{i}$, $x(i) \in\{0,1\}$, in its usual binary expansion. Define for $x, y \in \mathbb{Z}$,

$$
x \vee y:=z \text { where } z(i)=\max \{x(i), y(i)\}, \quad i \geq 0 .
$$

Also, define

$$
\begin{aligned}
\perp(x, y) & := \begin{cases}1 & \text { if } x(i) y(i)=0 \text { for all } i \\
0 & \text { otherwise }\end{cases} \\
x * y & :=\left\{\begin{array}{cc}
x+y & \text { if } \perp(x, y)=1 \\
* & \text { otherwise }
\end{array}\right. \\
\delta^{(*)} & :=0 \quad \text { (the zero map) } \\
B(x) & :=\{i \mid x(i)=1\}
\end{aligned}
$$

Fact 2.2.

$$
\begin{aligned}
\left(\begin{array}{c}
x+y \\
x \text { is odd }
\end{array}\right) & \Leftrightarrow \perp(x, y)=1 \\
& \Leftrightarrow x \vee y=x+y .
\end{aligned}
$$


This is a standard result in number theory (e.g., see [GKP89]). We remark that setting $p=0$ in (2.1) shows that $\delta^{(0)}$ is the identity operator, i.e., $\delta^{(0)} H=H$, a fact we will occasionally use.

Fact 2.3.

$$
\delta^{(p)} \circ \delta^{(q)}=\delta^{(p * q)}= \begin{cases}\delta^{(p+q)} & \text { if } \perp(p, q)=1, \\ 0 & \text { otherwise. }\end{cases}
$$

Proof. The proof is essentially the same as that of Fact 2.1, except that here we get $\left(\begin{array}{c}p+q \\ p\end{array}\right)$ instead of $\left(\begin{array}{c}2 p \\ p\end{array}\right)$. Fact 2.2 then implies the desired conclusion.

It also follows from Fact 2.3 that

$$
\delta^{(p)} \circ \delta^{(q)}=\delta^{(q)} \circ \delta^{(p)}
$$

$$
\text { If } p=\sum_{i} 2^{p_{i}}, p_{1}<p_{2}<\cdots<p_{r} \text { then } \delta^{(p)}=\delta^{\left(2^{p_{1}}\right)} \circ \delta^{\left(2^{p_{2}}\right)} \circ \cdots \circ \delta^{\left(2^{p_{r}}\right)} .
$$

Remark (2.9) already suggests the dependence of the properties of $\delta^{(p)}$ on the form of the binary expansion of $p$. Our first result (in the next section) will determine the kernel of $\delta^{(p)}$ when $|B(p)|=1$, i.e., $p=2^{t}$ for some $t \geq 0$.

3. THE KERNEL OF $\delta^{(a)}: a=2^{t}$

The main result of this section is the following.

Theorem 1. If $a=2^{t}, t \geq 0$, and $|V|=n \geq(k+1) a, k \geq 0$, and $G=$ $G^{(k)}(n)=\left(V, \chi_{G}\right) \in C_{k}$ then

$$
\delta^{(a)} G=0 \Leftrightarrow G=\delta^{(a)} F \quad \text { for some } F \in C_{k-a} .
$$

Remark. (3.10) asserts that the kernel of $\delta_{k}^{(a)}$ is just the image of $\delta_{k-a}^{(a)}$, i.e.,

$$
0 \rightarrow C_{k-a} \stackrel{\delta^{(a)}}{\rightarrow} C_{k} \stackrel{\delta^{(a)}}{\rightarrow} C_{k+a} \rightarrow 0
$$

is a short exact sequence.

At the end of this section, we give examples showing why some restriction on $n$ is necessary.

Proof. The proof will proceed by induction on $k$. We first consider the case $k=0$.

Considering the two different 0 -graphs $G_{0}^{(0)}$ and $G_{1}^{(0)}$, it is easy to see that only $G=G_{0}^{(0)}$ satisfies the hypothesis that $\delta^{(a)} G=0$ (since $\delta^{(a)} G_{1}^{(0)}$ has edge set $\left.\left(\begin{array}{c}V \\ a\end{array}\right)\right)$. However, $G_{0}^{(0)}=\delta^{(a)} F^{(-a)}$, since any such graph $F^{(-a)}$ is 0 by definition. Therefore (3.10) holds for $k=0$.

To quell a potentially uneasy feeling about starting the induction at such a trivial level, we next give a direct proof of (3.10) for $k=1$. Let $G=(V, E)$ be a 1-graph with $n \geq 2 a$ and assume $\delta^{(a)} G=0$.

First, suppose $a=1$. Thus, $\delta^{(1)} G=0$ so that every pair $\{x, y\} \subset V$ contains an even number of elements of $E$. This implies that either $E=\varnothing$, in which case $G=\delta^{(1)} G_{0}^{(0)}$, or $E=V$, in which case $G=\delta^{(1)} G_{1}^{(0)}$, which shows that (3.10) holds in this case. 
Now, suppose $a=2^{t}>1$. If $E=\varnothing$ then $G=0=\delta^{(a)} F^{(1-a)}$ since by definition $F^{(1-a)}=0$ for $a>1$, and (3.10) holds. If $E=V$ then for $|S|=a+1, \chi_{\delta^{(a)} G}(S)=|S \cap E|=|S| \equiv 1(\bmod 2)$, which contradicts the hypothesis that $\delta^{(a)} G=0$. So assume $\varnothing \neq E \neq V$. In this case, however, it is impossible for $|S \cap E| \equiv 0(\bmod 2)$ for all $S \subset V$ with $|S|=a+1$ (which is implied by $\delta^{(a)} G=0$ ). Thus, (3.10) holds for $k=1$.

We now assume that (3.10) holds for all values less than some fixed $k \geq 2$, and $G$ is $k$-graph on $n \geq a(k+1)$ vertices satisfying $\delta^{(a)} G=0$.

Let $A \subset V$ be a fixed (arbitrary) subset of $V$ with $|A|=a=2^{t}$, and let

$$
\bar{V}:=V \backslash A, \quad G^{\prime}=G+\delta^{(a)} G(A),
$$

where $G(A)$ denotes the $(k-a)$-graph $\left(\bar{V}, \chi_{G(A)}\right)$ defined by

$$
\chi_{G(A)}(Y)=\chi_{G}(Y \cup A) \text { for } Y \in\left(\begin{array}{c}
\bar{V} \\
k-a
\end{array}\right) .
$$

Thus,

$$
\delta^{(a)} G^{\prime}=\delta^{(a)} G+\delta^{(a)} \delta^{(a)} G(A)=\delta^{(a)} G=0 .
$$

If we prove that $G^{\prime}=\delta^{(a)} F^{\prime}$ then we have

$$
\begin{aligned}
G & =G^{\prime}+\delta^{(a)} G(A) \quad(\text { over } \mathbb{Z} / 2) \\
& =\delta^{(a)} F^{\prime}+\delta^{(a)} G(A)=\delta^{(a)}\left(F^{\prime}+G(A)\right),
\end{aligned}
$$

and (3.10) holds.

Note that no edge of $G^{\prime}$ contains $A$, since any such edge $X$ of $G$ has $X \backslash A$ as an edge of $G(A)$, and so is cancelled in $G^{\prime}=G+\delta^{(a)} G(A)$.

So we may henceforth assume that this normalization has been made, and therefore that

$$
G \text { has no edge containing } A \text {. }
$$

Observe that for all $B \subset A, X$ is an edge of $G(B)$ if and only if $B \cup X$ is an edge of $G$.

Define $\bar{\delta}^{(a)}:=\left.\delta^{(a)}\right|_{\bar{V}}$. That is,

$$
\bar{\delta}^{(a)} F=\sum_{Y \in\left(\begin{array}{c}
\overline{\bar{V}} \\
k
\end{array}\right)} \sum_{X \in\left(\begin{array}{c}
Y \\
k-a
\end{array}\right)} \chi_{F}(X) Y .
$$

Fact 3.1. For $\varnothing \neq B \subseteq A$,

$$
\bar{\delta}^{(a)} G(B)=\sum_{C \subsetneq B} \bar{\delta}^{(a-b+c)} G(C)
$$

where $b=|B|, c=|C|$.

Note that for $B=A,(3.13)$ gives

$$
\bar{\delta}^{(a)} G(A)=\bar{\delta}^{(a)} 0=0=\sum_{C \subsetneq A} \bar{\delta}^{(c)} G(C) .
$$


This implies that if $\bar{G}:=\left.G\right|_{\bar{V}}$ then by (3.11),

$$
\bar{G}=\sum_{\varnothing \neq C \subsetneq A} \bar{\delta}^{(c)} G(C) .
$$

Proof of Fact 3.1. Consider $W=B \cup \bar{W}$ where $W \subset \bar{V},|\bar{W}|=k+a-b$. Observe that all edges of $G$ in $B \cup \bar{W}$ are of the form $C \cup Z$, where $C \subseteq B$ and $Z \subseteq \bar{W}$. Thus, we have

$$
\begin{aligned}
0 & =\chi_{\delta^{(a)} G}(W) \quad\left(\text { since } \delta^{(a)} G=0\right) \\
& =\chi_{\delta^{(a)} G}(B \cup \bar{W}) \\
& =\sum_{C \subseteq B} \chi_{\delta^{(a-b+c)} G(C)}(\bar{W}) \\
& =\sum_{C \subseteq B} \chi_{\bar{\delta}^{(a-b+c)} G(C)}(\bar{W}) \quad(\text { since } \bar{W} \subseteq \bar{V}) .
\end{aligned}
$$

Therefore,

$$
\begin{aligned}
\chi_{\bar{\delta}^{(a)} G(B)}(\bar{W}) & =\sum_{C=B} \chi_{\bar{\delta}^{(a-b+c)} G(C)}(\bar{W}) \\
& =\sum_{C \varsubsetneqq B} \chi_{\bar{\delta}^{(a-b+c)} G(C)}(\bar{W}) \text { by }(3.16) .
\end{aligned}
$$

Since $\bar{W} \subseteq \bar{V}$ with $|\bar{W}|=k+a-b$ was arbitrary then we conclude

$$
\bar{\delta}^{(a)} G(B)=\sum_{C \subsetneq B} \bar{\delta}^{(a-b+c)} G(C)
$$

which proves Fact 3.1 .

Fact 3.2. There exist graphs $F_{C}$ for $\varnothing \neq C \subseteq A$ such that for all $B \subseteq A$, $B \neq \varnothing$, we have

$$
\bar{G}(B)=\sum_{c=1}^{b} \bar{\delta}^{(a-b+c)} F_{c}^{*}
$$

where

$$
F_{c}^{*}= \begin{cases}\sum_{\varnothing \neq C \subseteq B} F_{C} \quad \text { if } \perp(a-b, c)=0, \\ \sum_{\varnothing \neq C \nsubseteq B} F_{C} \quad \text { if } \perp(a-b, c)=1\end{cases}
$$

and $c=|C|$.

Proof. Induction on $b:=|B|$. Suppose $b=1$. Applying Fact 3.1, we have

$$
\bar{\delta}^{(a)} \bar{G}(B)=\bar{\delta}^{(a-1)} \bar{G}=0 .
$$

Since $\bar{G}(B)$ is a $(k-1)$-graph on $\bar{V}$ with $|\bar{V}|=n-a \geq(k+1) a-a=k a$ then we can apply Theorem 1 for $(k-1)$-graphs and conclude that $\bar{G}(B)=$ 
$\bar{\delta}^{(a)} F_{B}$ as required. This, in particular, defines $F_{B}$ for $|B|=1$. Next we assume that Fact 3.2 has been proved for all values less than some $b \geq 2$, and suppose $B \subseteq A$ has $|B|=b$.

From Fact 3.1 we have

$$
\begin{aligned}
\bar{\delta}^{(a)} \bar{G}(B) & =\sum_{C \subsetneq B} \bar{\delta}^{(a-b+c)} \bar{G}(C) \\
& =\bar{\delta}^{(a-b)} \bar{G}+\sum_{\varnothing \neq C \subsetneq B} \bar{\delta}^{(a-b+c)} \bar{G}(C) \\
& =\sum_{\substack{\varnothing \neq C \subsetneq A \\
c<b}} \perp(a-b, c) \bar{\delta}^{(a-b+c)} \bar{G}(C)+\sum_{\varnothing \neq C \subsetneq B} \bar{\delta}^{(a-b+c)} \bar{G}(C)
\end{aligned}
$$

since by (3.15), $\bar{G}=\sum_{\varnothing \neq C \subsetneq A} \bar{\delta}^{(c)} \bar{G}(C)$ and since $\perp(a-b, c)=0$ if $c \geq b$. Next, for $c<b$, we obtain

$$
\bar{\delta}^{(a-b+c)} \bar{G}(C)=\bar{\delta}^{(a-b+c)} \sum_{i=1}^{c} \bar{\delta}^{(a-c+i)} F_{i}^{*}
$$

by the induction hypothesis (since $c<b$ ). Therefore

$$
\bar{\delta}^{(a-b+c)} \bar{G}(C)=\bar{\delta}^{(a-b+c)} \bar{\delta}^{(a)} F_{C}
$$

by the definition of $F_{i}^{*}$ since for $i<c$ and $a-b+c<2^{t}$, if

$$
\perp(a-b+c, a-c+i)=1
$$

then we have $(a-b+c)+(a-c+i)=2 a-b+i<2^{t}=a$, i.e., $a<b$ which is a contradiction. (This implies that for $i<c$, we have $\perp(a-b+c, a-c+i)=$ 0.) Thus,

$$
\begin{aligned}
& \bar{\delta}^{(a)} \bar{G}(B)=\sum_{\substack{\varnothing \neq C \subset \subset A \\
c<b}} \perp(a-b, c) \bar{\delta}^{(a-b+c)} \bar{\delta}^{(a)} F_{C} \\
& +\sum_{\varnothing \neq C \subsetneq^{\prime} B} \bar{\delta}^{(a-b+c)} \bar{\delta}^{(a)} F_{C} \\
& =\bar{\delta}^{(a)} \sum_{c=1}^{b-1} \bar{\delta}^{(a-b+c)} F_{c}^{*}
\end{aligned}
$$

by definition of $F_{c}^{*}$ (where the two cases $\perp(a-b, c)=0$ and $\perp(a-b, c)$ $=1$ are considered separately).

Therefore, we can apply Theorem 1 for $(k-b)$-graphs and conclude that

$$
\bar{G}(B)=\sum_{c=1}^{b-1} \bar{\delta}^{(a-b+c)} F_{c}^{*}+\bar{\delta}^{(a)} F_{B}
$$


for some $F_{B}$ (this is the definition of $F_{B}$ ).

$$
\bar{G}(B)=\sum_{c=1}^{b} \bar{\delta}^{(a-b+c)} F_{c}^{*}
$$

as required, since $F_{b}^{*}=F_{B}$. This completes the induction step and Fact 3.2 is proved.

Fact 3.3. For $0 \leq c \leq b<a=2^{t}$, and $b \geq 2$

$$
\sum_{i=1}^{c}\left(\begin{array}{l}
b \\
i
\end{array}\right) \equiv\left(\begin{array}{c}
b-1 \\
c
\end{array}\right) \equiv\left(\begin{array}{c}
a-b+c \\
c
\end{array}\right) \equiv \perp(a-b, c)(\bmod 2)
$$

Proof.

$$
\sum_{i=0}^{c}\left(\begin{array}{l}
b \\
i
\end{array}\right) \equiv \sum_{i=0}^{c}\left(\left(\begin{array}{c}
b-1 \\
i
\end{array}\right)+\left(\begin{array}{c}
b-1 \\
i-1
\end{array}\right)\right)
$$

(since $b \geq 2$ where $\left(\begin{array}{c}x \\ -1\end{array}\right):=0$ )

$$
\equiv\left(\begin{array}{c}
b-1 \\
c
\end{array}\right)+\left(\begin{array}{c}
b-1 \\
-1
\end{array}\right) \equiv\left(\begin{array}{c}
b-1 \\
c
\end{array}\right)(\bmod 2) \text {. }
$$

It remains to show (by Fact 2.2) that

$$
\left(\begin{array}{c}
b-1 \\
c
\end{array}\right) \equiv\left(\begin{array}{c}
a-b+c \\
c
\end{array}\right)(\bmod 2) .
$$

However, this follows easily by induction on $b$.

Definition. For $J \subseteq A, \varnothing \neq C \varsubsetneqq A$, define $F_{C}^{J}$ to be the graph having as its edges all sets of the form $J \cup X$ where $X$ is an edge of $F_{C}$, the graph defined in Fact 3.2.

Thus, for $J=\varnothing, F_{C}^{\varnothing}=F_{C}$. Also define $j:=|J|$.

Fact 3.4.

$$
G=\sum_{\substack{\varnothing \neq C \subsetneq A \\ C \neq J \neq A \\ j \leq c}} \delta^{(a+c-j)} F_{C}^{J}
$$

Proof. We first consider an arbitrary set $X \in\left(\begin{array}{l}\bar{V} \\ k\end{array}\right)$.

Then, since $\bar{G}=\sum_{\varnothing \neq C \subset_{\ddagger}} \bar{\delta}^{(c)} \bar{G}(C)$,

$$
\begin{aligned}
\chi_{G}(X) & =\chi_{\bar{G}}(X)=\chi_{\sum_{\varnothing \neq C \subsetneq A} \bar{\delta}^{(c)} \bar{G}(C)}(X) \\
& =\chi_{\sum_{\varnothing \neq C \subsetneq A} \bar{\delta}^{(c)} \sum_{i=1}^{c} \bar{\delta}^{(a-c+i)} F_{i}^{*}}(X) \\
& =\chi_{\sum_{\varnothing \neq C \subsetneq A} \bar{\delta}^{(a)} \bar{\delta}^{(c)} F_{C}}(X)
\end{aligned}
$$


because $\perp(c, a-c+i)=0$ for $i<c$. On the other hand, for the right-hand side of (3.20),

$$
X \text { is an edge of } \sum_{\substack{\varnothing \neq C \subsetneq \subsetneq A \\ C \neq J \neq \\ j \leq c}} \delta^{(a+c-j)} F_{C}^{J}
$$

if and only if

$$
X \text { is an edge of } \sum_{\varnothing \neq C \varsubsetneqq A} \delta^{(a+c)} F_{C}
$$

if and only if (by (3.21)) $X$ is an edge of $G$.

Next, suppose $X=B \cup \bar{X}$ where $\varnothing \neq B \subseteq A, \bar{X} \subset \bar{V},|X|=k$. Assume first that $B=A$. Then by (3.11) we have $\chi_{G}(X)=0$. On the right-hand side of (3.20), for each edge $Y$ of $F_{C}$ in $\bar{X}$, the number of $J$ for which $J \cup Y$ is in $X$ is exactly

$$
\begin{aligned}
&\left(\begin{array}{l}
a \\
0
\end{array}\right)+\left(\begin{array}{l}
a \\
1
\end{array}\right)+\cdots+\left(\begin{array}{l}
a \\
c
\end{array}\right)-1 \\
&(\text { the }-1 \text { coming from the requirement that } J \neq C) \\
& \equiv\left(\begin{array}{c}
a-1 \\
c
\end{array}\right)-1 \equiv 0(\bmod 2)
\end{aligned}
$$

by Fact 3.3, since all the digits in the binary expression of $a-1$ are 1 .

Now, suppose $\varnothing \neq B \varsubsetneqq A$. Then

$$
\chi_{G}(B \cup \bar{X})=\chi_{\bar{G}(B)}(\bar{X})=\chi_{\sum_{c=1}^{b} \bar{\delta}^{(a-b+c)} F_{c}^{*}}(\bar{X})
$$

On the other hand, consider the right-hand side of (3.20) for $B \cup \bar{X}$, i.e.,

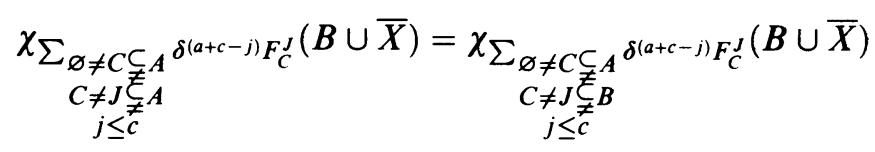

since any edge of $F_{C}^{J}$ by definition contains $J$. For a fixed $C \subseteq B$, the number of $J$ so that $j \leq c$ and $C \neq J \subseteq B$ is just

$$
\lambda:=\left(\begin{array}{l}
b \\
0
\end{array}\right)+\left(\begin{array}{l}
b \\
1
\end{array}\right)+\cdots+\left(\begin{array}{l}
b \\
c
\end{array}\right)-1 \quad(\text { since } J \neq C),
$$

while for $C \nsubseteq B$, the corresponding number is just $\lambda+1$. By Fact 3.3.

$$
\lambda+1 \equiv\left(\begin{array}{c}
b-1 \\
c
\end{array}\right) \equiv\left(\begin{array}{c}
a-b+c \\
c
\end{array}\right) \equiv \perp(a-b, c) \quad(\bmod 2) .
$$


Continuing (3.23) we get

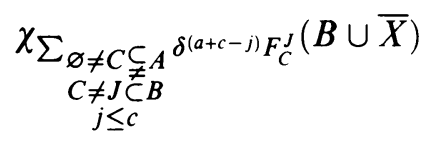

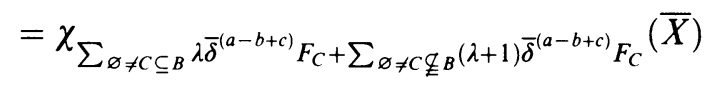

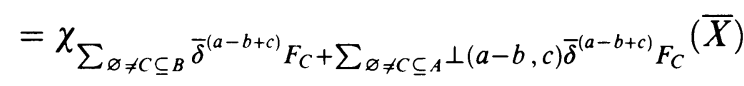

$$
\begin{aligned}
& =\chi_{\bar{\delta}^{(a-b+c)}}\left(\sum_{\varnothing \neq c \subseteq B} F_{C}+\sum_{\varnothing \neq c \subseteq A} \perp(a-b, c) F_{C}\right)(\bar{X}) \\
& =\chi_{\sum_{c=1}^{b} \bar{\delta}^{(a-b+c)} F_{c}^{*}}(\bar{X}) \text { by the definition of } F_{c}^{*} \\
& =\chi_{G}(B \cup \bar{X}) \text { by }(3.22) \text {. }
\end{aligned}
$$

This completes the proof of Fact 3.4.

To finish the proof of Theorem 1, observe by Fact 3.4 that in fact

$$
G=\delta^{(a)}\left(\sum_{\substack{\varnothing \neq C \subsetneq A \\ C \neq J \neq A \\ j \leq c}} \delta^{(c-j)} F_{c}^{J}\right)
$$

since $0 \leq c-j<a=2^{t}$ implies $\perp(a, c-j)=1$.

The other direction of $(3.10)$, namely that

$$
G=\delta^{(a)} F \Rightarrow \delta^{(a)} G=\delta^{(a)} \delta^{(a)} F=0
$$

follows from Fact 2.1. This completes the proof of Theorem 1.

There are a variety of examples known to show that some size restriction on $n$ is necessary in order for the conclusion of Theorem 1 be valid. One such family of examples is the following. Define

$$
V=\left\{x_{1}, x_{2}\right\} \cup \mathbb{Z} / 2^{t+1} \text { for } t \geq 1,
$$

$G=G^{\left(2^{t}+1\right)}=(V, E)$ with the edge set

$$
E=\left\{x_{j} \cup\left\{i+1, \ldots, i+2^{t}\right\} \mid j=1,2 \text { and } i \in \mathbb{Z} / 2^{t+1}\right\}
$$

Thus, $G$ has $2^{t+2}$ edges, each of which is a $\left(2^{t}+1\right)$-set in $V$. A simple calculation shows that $\delta^{\left(2^{t}\right)} G=0$. However, $G \neq \delta^{\left(2^{t}\right)} G^{(1)}$ for any 1-graph $G^{(1)}$ since no $\left(2^{t}+1\right)$-graph of the form $\delta^{\left(2^{t}\right)} G^{(1)}$ can have exactly $2^{t+2}$ edges.

4. THE KERNEL OF $\delta^{(a)}$ : GENERAL $a$

In this section we complete our analysis of $\operatorname{ker} \delta(a)$. In order to do this, we require an auxiliary result, of interest in its own right. 
Theorem 2. Suppose $a_{1}, a_{2}, \ldots, a_{r} \geq 0$ and $G_{i}$ is $a\left(k-a_{i}\right)$-graph on $V$ with $|V|=n \geq \frac{1}{4}(k+1)^{2}$. Then

$$
\begin{aligned}
& \sum_{i=1}^{r} \delta^{\left(a_{i}\right)} G_{i}=0 \Leftrightarrow \text { there exist } K_{i j}=K_{j i} \text { such that } \\
& \delta^{\left(a_{i}\right)} G_{i}=\sum_{\substack{j \\
j \neq i}} \delta^{\left(a_{i} \vee a_{j}\right)} K_{i j} .
\end{aligned}
$$

Proof. The proof will be a multiple induction on $r, \sum_{i} a_{i}$ and $k$. The desired conclusion holds for $r=1$ by Theorem 1. Also, Theorem 2 is immediate for $k=0$, so we will always assume henceforth that $k \geq 1$. We will first require several facts which will be proved under our induction hypotheses.

Fact 4.1. Suppose $a \leq \max _{i} a_{i}$ and $G$ is a $k^{\prime}$-graph with $a+k^{\prime} \leq k$. Then

$$
\delta^{(a)} G=0 \Leftrightarrow G=\sum_{t \in B(a)} \delta^{\left(2^{t}\right)} F_{t} \quad \text { for some } F_{t} .
$$

Proof. The result is immediate for $|B(a)|=1$. Suppose $|B(a)|>1$. Let $u:=$ $\min \{t \mid t \in B(a)\}$. By Theorem 1 (since $n \geq \frac{1}{4}(k+1)^{2} \geq 2^{u}\left(k^{\prime}+a-2^{u}+1\right)$ ),

$$
\delta^{\left(a-2^{u}\right)} G=\delta^{\left(2^{u}\right)} F \text { for some } F,
$$

i.e.,

$$
\delta^{\left(a-2^{u}\right)} G+\delta^{\left(2^{u}\right)} F=0 .
$$

Now by induction (since $k^{\prime}+a-2^{u}<k$ ), (4.26) implies

$$
\delta^{\left(a-2^{u}\right)} G=\delta^{(a)} F_{u},
$$

i.e.,

$$
\delta^{\left(a-2^{u}\right)}\left(G+\delta^{\left(2^{u}\right)} F_{u}\right)=0 .
$$

By induction within the proof of Fact 4.1, we have

$$
G+\delta^{\left(2^{u}\right)} F_{u}=\sum_{t \in B\left(a-2^{u}\right)} \delta^{\left(2^{t}\right)} F_{t} .
$$

Therefore,

$$
G=\sum_{t \in B(a)} \delta^{\left(2^{t}\right)} F_{t}
$$

as required.

Fact 4.2. Suppose all the edges of $G$ and $F_{i}, 1 \leq i \leq r$, are in $\bar{V}:=V \backslash\{v\}$. If

$$
\bar{G}=\left.G\right|_{\bar{V}}=\sum_{i=1}^{r} \bar{\delta}^{\left(b_{i}\right)} F_{i}
$$

then

$$
G=\sum_{i=1}^{r} \delta^{\left(b_{i}\right)} F_{i}+\sum_{i=1}^{r} \delta^{\left(b_{i}-1\right)} F_{i}^{+}
$$


where $F_{i}^{+}$has edge set $\left\{e \cup\{v\} \mid e \in E\left(F_{i}\right)\right\}$, and $\bar{\delta}^{(b)}:=\left.\delta^{(b)}\right|_{\bar{V}}$ as given in (3.12).

Proof. It is straightforward to verify (4.28) for the two possible cases, namely, edges which belong to $\bar{V}$, and edges of the form $e^{\prime} \cup\{v\}, e^{\prime} \subseteq \bar{V}$.

Fact 4.3. Assume $a \leq a_{i}$ for some $i$, and $a$ is even. Suppose $\bar{\delta}^{(a)} F=\bar{\delta}^{(a \vee b)} G$, and all edges of $F$ and $G$ are contained in $\bar{V}$. Then

$$
\delta^{(a+1)} F= \begin{cases}\delta^{(a \vee b+1)} G & \text { if } b \text { is even } \\ \delta^{(a \vee b)} G^{+} & \text {if } b \text { is odd. }\end{cases}
$$

Proof. $\bar{\delta}^{(a)} F=\bar{\delta}^{(a \vee b)} G$ implies $\bar{F}=\bar{\delta}^{(a \vee b-a)} G+K^{\prime}$ where $K^{\prime} \in \operatorname{ker} \bar{\delta}^{(a)}$. Thus, by Fact 4.1, we have

$$
\bar{F}=\bar{\delta}^{(a \vee b-a)} G+\sum_{t \in B(a)} \bar{\delta}^{\left(2^{t}\right)} K_{t}
$$

By Fact 4.2, we have

$$
F=\delta^{(a \vee b-a)} G+\sum_{t \in B(a)} \delta^{\left(2^{t}\right)} K_{t}+\delta^{(a \vee b-a-1)} G^{+}+\sum_{t \in B(a)} \delta^{\left(2^{t}-1\right)} K_{t}^{+}
$$

Thus,

$$
\begin{aligned}
\delta^{(a+1)} F= & \delta^{(a+1) *(a \vee b-a)} G+\sum_{t \in B(a)} \delta^{(a+1) * 2^{t}} K_{t} \\
& +\delta^{(a+1) *(a \vee b-a-1)} G^{+}+\sum_{t \in B(a)} \delta^{(a+1) *\left(2^{t}-1\right)} K_{t}^{+} \\
= & \delta^{(a+1) *(a \vee b-a)} G+\delta^{(a+1) *(a \vee b-a-1)} G^{+}
\end{aligned}
$$

We consider two cases:

Case 1: $b$ is even. Therefore

$$
\delta^{(a+1)} F=\delta^{(a+1) *(a \vee b-a)} G=\delta^{(a \vee b+1)} G .
$$

Case 2: $b$ is odd. Therefore

$$
\delta^{(a+1)} F=\delta^{(a+1) *(a \vee b-a-1)} G^{+}=\delta^{(a \vee b)} G^{+}
$$

as required. This proves Fact 4.3.

Our next step will be to "normalize" the statement of Theorem 2 . We first claim that it is enough to prove Theorem 2 in the case that all the $a_{i}$ are distinct. To see this, assume Theorem 2 holds in this case, and suppose $a_{i}=a_{j}$ for some $i<j$. Thus, by hypothesis

$$
\delta^{\left(a_{i}\right)}\left(G_{i}+G_{j}\right)+\sum_{l \neq i, j} \delta^{\left(a_{l}\right)} G_{l}=0 .
$$

By induction (on the number of summands), we can find $W_{u, v}$ for $u, v \neq j$ so that

$$
\delta^{\left(a_{l}\right)} G_{l}=\sum_{u \neq l} \delta^{\left(a_{u} \vee a_{l}\right)} W_{u, l}
$$


and

$$
\delta^{\left(a_{i}\right)}\left(G_{i}+G_{j}\right)=\sum_{u \neq i, j} \delta^{\left(a_{i} \vee a_{u}\right)} W_{i, u}
$$

Now define

$$
K_{m, l}= \begin{cases}W_{m, l} & \text { if } m, l \neq j, \\ G_{j} & \text { if }\{m, l\}=\{i, j\}, \\ 0 & \text { if }\{m, l\}=\{u, j\}, u \neq i .\end{cases}
$$

It is easily checked that (4.26) holds with these choices.

Next, we show that in fact, it suffices to prove Theorem 2 for the case that all the $a_{i}$ are distinct powers of 2 . For, suppose $a_{1}=2^{t}+b$ where $t \in B\left(a_{1}\right)$ and $b>0$. Then, by hypothesis,

$$
\delta^{\left(2^{t}\right)}\left(\delta^{(b)} G_{1}\right)+\sum_{i>1} \delta^{\left(a_{i}\right)} G_{i}=0
$$

By induction (on $\sum_{i} a_{i}$ ), there exist $W_{i j}$ such that

$$
\delta^{\left(2^{t}\right)}\left(\delta^{(b)} G_{1}\right)=\sum_{i>1} \delta^{\left(2^{t} \vee a_{i}\right)} W_{1 i}
$$

and for $i>1$,

$$
\delta^{\left(a_{i}\right)} G_{i}=\sum_{j \neq i, 1} \delta^{\left(a_{i} \vee a_{j}\right)} W_{i j}+\delta^{\left(2^{t} \vee a_{i}\right)} W_{1 i}
$$

By (4.31),

$$
\delta^{(b)} G_{1}=\sum_{i>1} \delta^{\left(2^{t} \vee a_{i}-2^{t}\right)} W_{1 i}+\delta^{\left(2^{t}\right)} K
$$

for some $K$. We now apply the induction hypothesis of Theorem 2 (for a smaller value of $k$, namely $k-2 t$ ), and conclude there exist $U_{i j}, 0 \leq i<j \leq$ $r$, with

$$
\delta^{(b)} G_{1}=\sum_{i>1} \delta^{\left(2^{t} \vee a_{i}-2^{t}\right) \vee b} U_{1 i}+\delta^{\left(b \vee 2^{t}\right)} U_{01}
$$

and for $i>1$,

$$
\delta^{\left(2^{t} \vee a_{i}-2^{t}\right)} W_{1 i}=\sum_{\substack{j \\ j \neq i, 1}} \delta^{\left(2^{t} \vee a_{i} \vee a_{j}-2^{t}\right)} U_{i j}+\delta^{\left(2^{t} \vee a_{i}-2^{t}\right) \vee b} U_{1 i}+\delta^{\left(2^{t} \vee a_{i}\right)} U_{0 i}
$$

Therefore,

$$
\delta^{\left(a_{1}\right)} G_{1}=\sum_{i>1} \delta^{\left(a_{i} \vee a_{1}\right)} U_{1 i}
$$

and for $i>1$ (by (4.32)),

$$
\begin{aligned}
\delta^{\left(a_{i}\right)} G_{i} & =\sum_{j \neq i, 1} \delta^{\left(a_{i} \vee a_{j}\right)} W_{i j}+\delta^{\left(2^{l} \vee a_{i}\right)} W_{1 i} \\
& =\sum_{j \neq i, 1} \delta^{\left(a_{i} \vee a_{j}\right)} W_{i j}+\sum_{j \neq i, 1} \delta^{\left(2^{t} \vee a_{i} \vee a_{j}\right)} U_{i j}+\delta^{\left(a_{i} \vee a_{1}\right)} U_{1 i} .
\end{aligned}
$$


So, if we choose

$$
K_{i j}= \begin{cases}W_{i j}+\delta^{\left(2^{t}\right)} U_{i j} & \text { if } i, j \neq 1, \\ U_{1 i} & \text { if } i>1, j=1,\end{cases}
$$

then (4.26) is easily verified.

Finally, suppose some $a_{i}$ vanishes, say $a_{1}=0$. Then by choosing $K_{i j}=G_{i}$ if $i=1, j>1$, and $K_{i j}=0$ otherwise, the implication $(4.25) \Rightarrow(4.26)$ is immediate.

Thus, we may assume in the proof of Theorem 2 that $a_{i}=2^{b_{i}}$ with $0 \leq b_{1}<$ $b_{2}<\cdots<b_{r}$. We now return to the main line of the proof.

Define for $1 \leq i \leq r$,

$$
F_{i}^{\prime}:=G_{i}(v), \quad F_{i}=G_{i}+\delta^{(1)} F_{i}^{\prime}
$$

where $v$ is an arbitrary (fixed) vertex of $V$. It is easy to see that $F_{i}$ and $F_{i}^{\prime}$ have edge sets entirely contained in $\bar{V}:=V \backslash\{v\}$, and

$$
G_{i}=F_{i}+\delta^{(1)} F_{i}^{\prime}
$$

Hence,

$$
\sum_{i} \delta^{\left(a_{i}\right)} G_{i}=0
$$

implies

$$
\sum_{i} \delta^{\left(a_{i}\right)} F_{i}+\sum_{i} \delta^{\left(a_{i}+1\right)} F_{i}^{\prime}=0 .
$$

We first apply (4.35) to edges of the form $\bar{X} \cup\{v\}$ for $\bar{X} \in\left(\begin{array}{c}\bar{V} \\ k-1\end{array}\right)$. Thus,

$$
\sum_{i} \bar{\delta}^{\left(a_{i}-1\right)} F_{i}+\sum_{i} \bar{\delta}^{\left(a_{i}\right)} F_{i}^{\prime}=0
$$

We consider two cases.

Case 1. $b_{1}=0$. Thus, $a_{1}=1$ and $\delta^{\left(a_{1}\right)} G_{1}=\delta^{(1)} G_{1}=\delta^{(1)} F_{1}$ (since we can assume $\left.F_{1}^{\prime}=0\right)$. Applying $\bar{\delta}^{(1)}$ to $(4.36)$, we have

$$
\bar{\delta}^{(1)} F_{1}+\sum_{i>1} \bar{\delta}^{\left(a_{i}+1\right)} F_{i}^{\prime}=0 .
$$

Together with (4.35) we find

$$
\sum_{i>1} \bar{\delta}^{\left(a_{i}\right)} F_{i}=0
$$

We can now apply Theorem 2 since (4.38) involves $r-1$ summands. This implies that there exist $W_{i, j}, i, j>1$, so that for $i>1$,

$$
\bar{\delta}^{\left(a_{i}\right)} F_{i}=\sum_{\substack{j \\ j \neq 1, i}} \bar{\delta}^{\left(a_{i} \vee a_{j}\right)} W_{i j}
$$


Hence, by Theorem 1 (since $a_{i}=2^{b_{i}}$ ),

$$
\bar{F}_{i}:=\left.F_{i}\right|_{V}=\sum_{\substack{j \\ j \neq 1, i}} \bar{\delta}^{\left(a_{i} \vee a_{j}-a_{i}\right)} W_{i j}+\bar{\delta}^{\left(a_{i}\right)} K_{i}
$$

for some $K_{i}$. By Fact 4.2, we obtain

$$
F_{i}=\sum_{\substack{j \\ j \neq 1, i}} \delta^{\left(a_{i} \vee a_{j}-a_{i}\right)} W_{i j}+\sum_{\substack{j \\ j \neq 1, i}} \delta^{\left(a_{i} \vee a_{j}-a_{i}-1\right)} W_{i j}^{+}+\delta^{\left(a_{i}\right)} K_{i}+\delta^{\left(a_{i}-1\right)} K_{i}^{+} .
$$

Therefore, for $i>1$,

$$
\delta^{\left(a_{i}\right)} F_{i}=\sum_{\substack{j \\ j \neq 1, i}} \delta^{\left(a_{i} \vee a_{j}\right)} W_{i j}+\sum_{\substack{j \\ 1<j<i}} \delta^{\left(a_{i} \vee\left(a_{j}-1\right)\right)} W_{i j}^{+}+\delta^{\left(2 a_{i}-1\right)} K_{i}^{+},
$$

and

$$
\bar{\delta}^{\left(a_{i}-1\right)} F_{i}=\sum_{\substack{j \\ j>i}} \bar{\delta}^{\left(a_{j}+a_{i}-1\right)} W_{i j}+\bar{\delta}^{\left(2 a_{i}-1\right)} K_{i}
$$

Substituting into (4.36) we have

$$
\bar{F}_{1}+\sum_{\substack{i, j \\ 1<j<i}} \bar{\delta}^{\left(a_{i}+a_{j}-1\right)} W_{i j}+\sum_{i>1} \bar{\delta}^{\left(2 a_{i}-1\right)} K_{i}+\sum_{i>1} \bar{\delta}^{\left(a_{i}\right)} F_{i}^{\prime}=0
$$

i.e.,

$$
\bar{F}_{1}+\sum_{i>1} \bar{\delta}^{\left(a_{i}\right)}\left(\sum_{\substack{j \\ 1<j<i}} \bar{\delta}^{\left(a_{j}-1\right)} W_{i j}+\bar{\delta}^{\left(a_{i}-1\right)} K_{i}+\bar{F}_{i}^{\prime}\right)=0
$$

Now, define for $i>1$,

$$
L_{i}=\sum_{\substack{j \\ 1<j<i}} \bar{\delta}^{\left(a_{j}-1\right)} W_{i j}+\bar{\delta}^{\left(a_{i}-1\right)} K_{i}+\bar{F}_{i}^{\prime} .
$$

Then

$$
\bar{F}_{1}=\sum_{i>1} \bar{\delta}^{\left(a_{i}\right)} L_{i}
$$

and for $i>1$,

$$
\bar{F}_{i}^{\prime}=\sum_{\substack{j \\ 1<j<i}} \bar{\delta}^{\left(a_{j}-1\right)} W_{i j}+\bar{\delta}^{\left(a_{i}-1\right)} K_{i}+L_{i} .
$$

Using Fact 4.2, we obtain

$$
F_{1}=\sum_{i>1} \delta^{\left(a_{i}\right)} L_{i}+\sum_{i>1} \delta^{\left(a_{i}-1\right)} L_{i}^{+} .
$$


Also, for $i>1$,

$$
\bar{\delta}^{\left(a_{i}\right)} F_{i}^{\prime}=\sum_{\substack{j \\ 1<j<i}} \bar{\delta}^{\left(a_{j}+a_{i}-1\right)} W_{i j}+\bar{\delta}^{\left(2 a_{i}-1\right)} K_{i}+\bar{\delta}^{\left(a_{i}\right)} L_{i} .
$$

By Fact 4.3,

$$
\delta^{\left(a_{i}+1\right)} F_{i}^{\prime}=\sum_{\substack{j \\ 1<j<i}} \delta^{\left(a_{j}+a_{i}-1\right)} W_{i j}^{+}+\delta^{\left(2 a_{i}-1\right)} K_{i}^{+}+\delta^{\left(a_{i}+1\right)} L_{i} .
$$

Now, choose

$$
K_{i j}= \begin{cases}W_{i j} & \text { if } i, j>1 \\ L_{i} & \text { if } i>1, j=1\end{cases}
$$

Thus for $i>1$ we have (by (4.40) and (4.45)),

$$
\begin{aligned}
\delta^{\left(a_{i}\right)} G_{i}= & \delta^{\left(a_{i}\right)} F_{i}+\delta^{\left(a_{i}+1\right)} F_{i}^{\prime} \\
= & \sum_{\substack{j \\
j \neq 1, i}} \delta^{\left(a_{i} \vee a_{j}\right)} W_{i j}+\sum_{\substack{j \\
1<j<i}} \delta^{\left(a_{i} \vee\left(a_{j}-1\right)\right)} W_{i j}^{+} \\
& +\delta^{\left(2 a_{i}-1\right)} K_{i}^{+}+\sum_{\substack{j \\
1<j<i}} \delta^{\left(a_{i}+a_{j}-1\right)} W_{i j}^{+} \\
& +\delta^{\left(2 a_{i}-1\right)} K_{i}^{+}+\delta^{\left(a_{i}+1\right)} L_{i} \\
= & \sum_{\substack{j \neq i \\
j \neq i}} \delta^{\left(a_{i} \vee a_{j}\right)} K_{i j} .
\end{aligned}
$$

Finally, by (4.44) we get

$$
\delta^{\left(a_{1}\right)} G=\delta^{(1)} F_{1}=\sum_{i>1} \delta^{\left(a_{i}+1\right)} L_{i}=\sum_{i>1} \delta^{\left(a_{i}+a_{1}\right)} K_{1 i}
$$

and the proof for Case 1 is complete.

Case 2. $a_{1}=2^{b_{1}}>1$. We apply $\bar{\delta}^{(1)}$ to (4.36). Thus,

$$
\sum_{i} \bar{\delta}^{\left(a_{i}+1\right)} F_{i}^{\prime}=0
$$

Hence, by (4.35) (restricted to $\bar{V}$ ), we have

$$
\sum_{i} \bar{\delta}^{\left(a_{i}\right)} F_{i}=0
$$

Now, rearranging (4.36), we obtain

$$
\sum_{i} \bar{\delta}^{\left(a_{i}\right)} F_{i}^{\prime}=\sum_{i} \bar{\delta}^{\left(a_{i}-1\right)} F_{i}=\bar{\delta}^{\left(a_{1}-1\right)}\left(\sum_{i} \bar{\delta}^{\left(a_{i}-a_{1}\right)} F_{i}\right) .
$$


We next apply Theorem 2 to (4.48) (which has a smaller value of $k$ ). Thus, there exist $W_{i j}, 0 \leq i<j \leq r$, such that

$$
\bar{\delta}^{\left(a_{1}-1\right)}\left(\sum_{i} \bar{\delta}^{\left(a_{i}-a_{1}\right)} F_{i}\right)=\sum_{i} \bar{\delta}^{\left(a_{i}+a_{1}-1\right)} W_{i 0},
$$

and

$$
\bar{\delta}^{\left(a_{i}\right)} F_{i}^{\prime}=\sum_{j \neq i} \bar{\delta}^{\left(a_{i}+a_{j}\right)} W_{i j}+\bar{\delta}^{\left(a_{i}+a_{1}-1\right)} W_{i 0} .
$$

Hence, by Fact 4.3,

$$
\delta^{\left(a_{i}+1\right)} F_{i}^{\prime}=\sum_{j \neq i} \delta^{\left(a_{i}+a_{j}+1\right)} W_{i j}+\delta^{\left(a_{i}+a_{1}-1\right)} W_{i 0}^{+} .
$$

Applying Fact 4.1 to (4.49), we have

$$
\sum_{i} \bar{\delta}^{\left(a_{i}-a_{1}\right)} F_{i}=\sum_{i=1}^{r} \bar{\delta}^{\left(a_{i}\right)} W_{i 0}+\sum_{t \in B\left(a_{1}-1\right)} \bar{\delta}^{\left(2^{t}\right)} K_{t}
$$

for some $K_{t}$. Applying $\bar{\delta}^{\left(a_{1}\right)}$ to both sides of (4.52), we obtain

$$
\bar{\delta}^{\left(a_{1}\right)} F_{1}=\sum_{i>1} \bar{\delta}^{\left(a_{i}+a_{1}\right)} W_{i 0}+\sum_{t<b_{1}} \bar{\delta}^{\left(2^{t}+a_{1}\right)} K_{t} .
$$

Using (4.47), we have

$$
\sum_{i>1} \bar{\delta}^{\left(a_{i}\right)}\left(\delta^{\left(a_{1}\right)} W_{i 0}+F_{i}\right)+\sum_{t<b_{1}} \bar{\delta}^{\left(2^{t}\right)}\left(\delta^{\left(a_{1}\right)} K_{t}\right)=0 .
$$

Since $\sum_{t<b_{1}} 2^{t}<a_{1}$, we can apply the induction hypothesis of Theorem 2 . Therefore, there exist $X_{i j}, Y_{i t}, Y_{t, t^{\prime}}^{\prime}, 0 \leq t, t^{\prime}<b_{1}, i, j>1$, satisfying:

For $i>1$,

$$
\begin{gathered}
\bar{\delta}^{\left(a_{i}\right)}\left(\bar{\delta}^{\left(a_{1}\right)} W_{i 0}+F_{i}\right)=\sum_{\substack{j \\
j \neq 1, i}} \bar{\delta}^{\left(a_{i}+a_{j}\right)} X_{i j}+\sum_{t} \bar{\delta}^{\left(2^{t}+a_{i}\right)} Y_{i t}, \\
\bar{\delta}^{\left(2^{\prime}+a_{1}\right)} K_{t}=\sum_{i>1} \bar{\delta}^{\left(a_{i}+2^{t}\right)} Y_{i t}+\sum_{t^{\prime} \neq t} \bar{\delta}^{\left(2^{t}+2^{\left.t^{\prime}\right)}\right.} Y_{t t^{\prime}}^{\prime}
\end{gathered}
$$

This implies by Theorem 1 that

$$
\bar{\delta}^{\left(a_{1}\right)} K_{t}=\sum_{i>1} \bar{\delta}^{\left(a_{i}\right)} Y_{i t}+\sum_{t^{\prime} \neq t} \bar{\delta}^{\left(2^{t^{\prime}}\right)} Y_{t t^{\prime}}^{\prime}+\bar{\delta}^{\left(2^{t}\right)} J_{t}
$$

for some $J_{t}$. Applying the induction hypothesis of Theorem 2 to (4.57) (since $k$ is smaller), we have: 
For $i>1$,

$$
\bar{\delta}^{\left(a_{i}\right)} Y_{i t}=\sum_{j \neq i} \bar{\delta}^{\left(a_{i}+a_{j}\right)} Z_{i j}^{(t)}+\sum_{t^{\prime}} \bar{\delta}^{\left(a_{i}+2^{t^{\prime}}\right)} Z_{t t^{\prime}}^{\prime}
$$

for $Z_{i j}^{(t)}, Z_{t t^{\prime}}^{\prime}, 0 \leq t, t^{\prime}<b_{1}, 1<j$. Therefore,

$$
\bar{\delta}^{\left(a_{i}+2^{t}\right)} Y_{i t}=\sum_{j \neq 1, i} \bar{\delta}^{\left(a_{i}+a_{j}+2^{t}\right)} Z_{i j}^{(t)}+\sum_{t^{\prime} \neq t} \bar{\delta}^{\left(a_{i}+2^{t}+2^{t^{\prime}}\right)} Z_{t t^{\prime}}^{\prime}
$$

Substituting into (4.55) we obtain

$$
\bar{\delta}^{\left(a_{i}\right)}\left(\delta^{\left(a_{1}\right)} W_{i 0}+F_{i}\right)=\sum_{\substack{j \\ j \neq 1, i}} \bar{\delta}^{\left(a_{i}+a_{j}\right)} X_{i j}+\sum_{\substack{j, t \\ j \neq 1, i}} \bar{\delta}^{\left(a_{i}+a_{j}+2^{t}\right)} Z_{i j}^{(t)}
$$

Therefore, by Fact 4.1, we have

$$
\bar{F}_{i}=\bar{\delta}^{\left(a_{1}\right)} W_{i 0}+\sum_{\substack{j \\ j \neq 1, i}} \bar{\delta}^{\left(a_{j}\right)} X_{i j}+\sum_{\substack{j, t \\ j \neq 1, i}} \bar{\delta}^{\left(a_{j}+2^{t}\right)} Z_{i j}^{(t)}+\bar{\delta}^{\left(a_{i}\right)} T_{i}
$$

for some $T_{i}$.

Hence, by Fact 4.2,

$$
\begin{aligned}
F_{i}= & \delta^{\left(a_{1}\right)} W_{i 0}+\sum_{\substack{j \\
j \neq l, i}} \delta^{\left(a_{j}\right)} X_{i j}+\sum_{\substack{j, t \\
j \neq 1, i}} \delta^{\left(a_{j}+2^{t}\right)} Z_{i j}^{(t)}+\delta^{\left(a_{i}\right)} T_{i} \\
& +\delta^{\left(a_{1}-1\right)} W_{i 0}^{+}+\sum_{\substack{j \\
j \neq 1, i}} \delta^{\left(a_{j}-1\right)} X_{i j}^{+}+\sum_{\substack{j, t \\
j \neq 1, i}} \delta^{\left(a_{j}+2^{t}-1\right)} Z_{i j}^{(t)^{+}}+\delta^{\left(a_{i}-1\right)} T_{i}^{+} .
\end{aligned}
$$

Thus, for $i>1$,

$$
\begin{aligned}
\delta^{\left(a_{i}\right)} F_{i}= & \delta^{\left(a_{i}+a_{1}\right)} W_{i 0}+\sum_{j \neq 1, i} \delta^{\left(a_{i}+a_{j}\right)} X_{i j} \\
& +\sum_{\substack{j \neq 1, i \\
t \in B\left(a_{1}-1\right)}} \delta^{\left(a_{i}+a_{j}+2^{t}\right)} Z_{i j}^{(t)}+\delta^{\left(a_{i}+a_{1}-1\right)} W_{i 0}^{+} \\
& +\sum_{1<j<i} \delta^{\left(a_{i}+a_{j}-1\right)} X_{i j}^{+}+\sum_{\substack{j \neq 1, i \\
t \in B\left(a_{1}-1\right)}} \delta^{\left(a_{i}+a_{j}+2^{t}-1\right)} Z_{i j}^{(t)^{+}}+\delta^{\left(2 a_{i}-1\right)} T_{i}^{+} .
\end{aligned}
$$

Now, by (4.63) and (4.51), we have for $i>1$,

$$
\begin{aligned}
\delta^{\left(a_{i}\right)} G_{i}= & \delta^{\left(a_{i}\right)} F_{i}+\delta^{\left(a_{i}-1\right)} F_{i}^{\prime} \\
= & \sum_{j \neq i, 1} \delta^{\left(a_{i} \vee a_{j}\right)}\left(X_{i j}+\sum_{t \in B\left(a_{1}-1\right)} \delta^{\left(2^{t}\right)} Z_{i j}^{(t)}+\sum_{t \in B\left(a_{1}-1\right)} \delta^{\left(2^{t}-1\right)} Z_{i j}^{(t)^{+}}+\delta^{(1)} W_{i j}\right) \\
& +\delta^{\left(a_{i} \vee a_{1}\right)}\left(W_{i 0}+\delta^{(1)} W_{i 1}+\sum_{1<j<i} \delta^{\left(a_{j}-a_{1}-1\right)} X_{i j}^{+}+\delta^{\left(a_{i}-a_{1}-1\right)} T_{i}^{+}\right) .
\end{aligned}
$$


Now, choose

$$
H_{i j}=H_{j i}=\left\{\begin{array}{c}
X_{i j}+\delta^{(1)} W_{i j}+\sum_{t \in B\left(a_{1}-1\right)} \delta^{\left(2^{t}\right)} Z_{i j}^{(t)}+\sum_{t \in B\left(a_{1}-1\right)} \delta^{\left(2^{t}-1\right)} Z_{i j}^{(t)^{+}} \\
W_{i 0}+\delta^{(1)} W_{i 1}+\sum_{1<j<i} \delta^{\left(a_{j}-a_{1}-1\right)} X_{i j}^{+}+\delta^{\left(a_{i}-a_{1}-1\right)} T_{i}^{+} \\
\text {for } i, j>1, \\
\text { for } j=1 \text { and } i>1 .
\end{array}\right.
$$

Therefore,

$$
\delta^{\left(a_{i}\right)} G_{i}=\sum_{j \neq i} \delta^{\left(a_{i} \vee a_{j}\right)} K_{i j} \quad \text { for } i>1
$$

Since

$$
\delta^{\left(a_{1}\right)} G_{1}=\sum_{i>1} \delta^{\left(a_{i}\right)} G_{i}=\sum_{i>1} \delta^{\left(a_{i} \vee a_{1}\right)} K_{i 1}
$$

then the required $H_{i j}$ have been exhibited, i.e., (4.26) holds. This completes the induction step and the proof of Theorem 2 is complete.

We can now use Fact 4.1 (which holds for all $k$ ) to characterize the kernel of $\delta^{(a)}$ for general $a$.

Theorem 3. Suppose $G$ is a k-graph on $n \geq \frac{1}{4}(k+a+1)^{2}$ vertices. Then

$$
\delta^{(a)} G=0 \Leftrightarrow G=\sum_{t \in B(a)} \delta^{\left(2^{t}\right)} K_{t} \text { for some choice of } K_{t} \text { 's . }
$$

Proof. " $\Rightarrow$ " The lower bound (which is actually rather generous) comes from that of Theorem 2, since $\delta^{(a)} G$ is a $(k+a)$-graph. Fact 4.1 gives the desired implication.

" $\Leftarrow$ " Immediate, using Fact 2.3.

Perhaps one could characterize those $G$ satisfying $\delta^{(a)} G=0$ but with $G \neq$ $\sum_{t \in B(a)} \delta^{2^{t}} K_{t}$.

\section{THE COHOMOLOGY GROUPS $H_{k}^{p, q}$}

Given a portion of the (generalized) chain complex at $C_{k}$ :

$$
\cdots \rightarrow C_{k-p} \stackrel{\delta^{(p)}}{\rightarrow} C_{k} \stackrel{\delta^{(q)}}{\rightarrow} C_{k+q} \rightarrow \cdots,
$$

it is natural to ask about the cohomology group $H_{k}^{p, q}:=\operatorname{ker} \delta^{(q)} / \operatorname{im} \delta^{(p)}$. Here, we assume that $B(p) \cap B(q) \neq \varnothing$, i.e., the binary expansions of $p$ and $q$ share a common one, since otherwise we can have $\delta^{(p)}\left(\delta^{(q)}(\cdot)\right) \neq 0$, i.e., im $\delta^{(p)} \subsetneq$ $\operatorname{ker} \delta^{(q)}$, so that $H_{k}^{p, q}$ is not well defined. It is easy to see under this assumption that $H_{k}^{p, q} \cong(\mathbb{Z} / 2)^{d(p, q ; k)}$ where $d(p, q ; k)$ is the dimension of the quotient space $\operatorname{ker} \delta^{(q)} / \operatorname{im} \delta^{(p)}$ (with $\operatorname{ker} \delta^{(q)}$ and im $\delta^{(p)}$ considered as vector spaces over $\mathbb{Z} / 2$ ). Thus, we need to compute the dimensions of $\operatorname{ker} \delta^{(q)}$ and $\operatorname{im} \delta^{(p)}$. In order to do this, we need to introduce the following class of matrices $W=$ $W_{r, s}$. For a fixed $n$-set $V$, the rows and columns of $W$ are indexed by the sets $\left(\begin{array}{l}V \\ r\end{array}\right)$ and $\left(\begin{array}{l}V \\ r\end{array}\right)$, with $r \geq s$. For $X \in\left(\begin{array}{l}V \\ r\end{array}\right), Y \in\left(\begin{array}{l}V \\ s\end{array}\right)$, the $(X, Y)$ entry $W(X, Y)$ 
of $W$ is defined by

$$
W(X, Y)= \begin{cases}1 & \text { if } X \supseteq Y, \\ 0 & \text { otherwise. }\end{cases}
$$

These inclusion matrices occur commonly in algebraic combinatorics (e.g., see [K72, GJ73, GLL80, LR81, F90, Wi*]). What will be of interest to us is the mod 2 rank $w_{r, s}$ of $W$ (i.e., the rank of the integer matrix $W$ over $\mathbb{Z} / 2$ ). This was first determined by Linial and Rothschild [LR81]. Subsequently, Wilson [Wi*] determined the $(\bmod p)$ rank of $W$ for every prime $p$, and expressed the rank $w_{r, s}$ in a form which will be especially convenient for our purposes (a very elegant proof also appears in Frankl [F90]).

Theorem 4 (Wilson [Wi*]). For $0 \leq s \leq r \leq n-s$,

$$
w_{r, s}=\sum_{i}\left(\left(\begin{array}{c}
n \\
i
\end{array}\right)-\left(\begin{array}{c}
n \\
i-1
\end{array}\right)\right)
$$

summed over all $i$ such that $\left(\begin{array}{c}r-i \\ s-i\end{array}\right)$ is odd (where $\left(\begin{array}{c}n \\ -1\end{array}\right):=0$ ).

As an immediate consequence, we have

Fact 5.1. $\operatorname{dim}\left(\operatorname{im} \delta^{(p)}\right)=w_{k, k-p}$.

Our main effort will be in determining $\operatorname{dim}\left(\operatorname{ker} \delta^{(q)}\right)$. To begin, write $B(q)=$ $\left\{q_{1}<q_{2}<\cdots<q_{r}\right\}$, so that $q=\sum_{i=1}^{r} 2^{q_{i}}$. Form the matrix $W^{*}$ by concate-

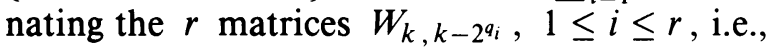

$$
W^{*}=W_{k, k-2^{q_{1}}} W_{k, k-2^{q_{2}}} \cdots W_{k, k-2^{q_{r}}} .
$$

It is not hard to see that by Theorem 3,

$$
\operatorname{dim}\left(\operatorname{ker} \delta^{(q)}\right)=\operatorname{rank}_{2} W^{*}
$$

(where rank $_{2}$ denotes the mod 2 rank). Now by inclusion-exclusion we have

$$
\begin{gathered}
\operatorname{rank}_{2} W^{*}=\sum_{i} w_{k, k-2^{q_{i}}}-\sum_{i<j} w_{k, k-2^{q_{i}}-2^{q_{j}}}+\sum_{i<j<l} w_{k, k-2^{q_{i}}-2^{q_{j}}-2^{q_{l}}} \\
-\cdots+(-1)^{r-1} w_{k, k-q} .
\end{gathered}
$$

Now, consider a typical term

$$
w_{k, k-c}=\sum_{i}\left(\left(\begin{array}{l}
n \\
i
\end{array}\right)-\left(\begin{array}{c}
n \\
i-1
\end{array}\right)\right)
$$

where $c=2^{s_{1}}+\cdots+2^{s_{l}}$, and the sum is taken over all $i$ such that $\left(\begin{array}{c}k-i \\ k-i-c\end{array}\right)$ is odd.

Changing the summation index in (5.67) from $i$ to $k-i$, and using Fact 2.2, we can rewrite (5.67) as

$$
w_{k, k-c}=\sum_{i}\left(\left(\begin{array}{c}
n \\
k-i
\end{array}\right)-\left(\begin{array}{c}
n \\
k-i-1
\end{array}\right)\right)
$$

summed over all $i$ such that $\left(\begin{array}{c}i \\ i-c\end{array}\right)=\left(\begin{array}{l}i \\ c\end{array}\right)$ is odd, i.e., such that $\left\{s_{1}, \ldots, s_{l}\right\}=$ $B(c) \subseteq B(i)$ (by Fact 2.2). Of course, for all our c's,

$$
B(c) \subseteq B(q)
$$


Thus,

$$
B(c) \subseteq B(i) \cap B(q)
$$

Letting $\{k-i\}$ denote $\left(\begin{array}{c}n \\ k-i\end{array}\right)-\left(\begin{array}{c}\cdot n \\ k-i-1\end{array}\right)$, we now count how often $\{k-i\}$ occurs in the various terms in (5.66), of which (5.67) is typical. Let $t:=|B(i) \cap B(q)|$. If $t=0$ then no $c$ 's contribute to the $\{k-i\}$ count, so suppose $t>0$. In this case, there are exactly $\left(\begin{array}{l}t \\ l\end{array}\right)$ different $c$ 's with $B(c) \subseteq B(i) \cap B(q),|B(c)|=l$, for which $\{k-i\}$ occurs in $w_{k, k-c}$. More exactly, it occurs with the sign $(-1)^{l-1}$, which comes from the corresponding $l$-tuple sum in (5.66). Therefore, the total contribution of $\{k-i\}$ in $(5.66)$ is just

$$
\sum_{l \geq 1}(-1)^{l-1}\left(\begin{array}{l}
t \\
l
\end{array}\right)=1 \text { if } t>0
$$

and 0 if $t=0$.

In particular, we obtain

$$
\operatorname{rank}_{2} W^{*}=\sum_{i}\left(\left(\begin{array}{c}
n \\
k-i
\end{array}\right)-\left(\begin{array}{c}
n \\
k-i-1
\end{array}\right)\right)
$$

summed over all $i$ such that $B(q) \cap B(i) \neq \varnothing$.

By Fact 5.1, we need to express $w_{k, k-p}$ in a similar form. This is given by (5.68):

$$
w_{k, k-p}=\sum_{i}\left(\left(\begin{array}{c}
n \\
k-i
\end{array}\right)-\left(\begin{array}{c}
n \\
k-i-1
\end{array}\right)\right)
$$

summed over all $i$ with $B(p) \subseteq B(i)$.

We can now put everything together for the main result of this section.

Theorem 5. When $B(p) \cap B(q) \neq \varnothing$, and $n \geq \frac{1}{4}(k+q+1)^{2}$ then

$$
H_{k}^{p, q}=\operatorname{ker} \delta^{(q)} / \operatorname{im} \delta^{(p)} \cong(\mathbb{Z} / 2)^{d(p, q ; k)}
$$

where

$$
d(p, q ; k)=\sum_{i}\left(\left(\begin{array}{c}
n \\
k-i
\end{array}\right)-\left(\begin{array}{c}
n \\
k-i-1
\end{array}\right)\right)
$$

summed over all $i$ such that

$$
B(p) \nsubseteq B(i) \text { and } B(q) \cap B(i) \neq \varnothing .
$$

Proof. Since

$$
d(p, q ; k)=\operatorname{dim}\left(\operatorname{ker} \delta^{(q)}\right)-\operatorname{dim}\left(\operatorname{im} \delta^{(p)}\right)
$$

then by (5.70) and (5.71), we simply have to keep track of the coefficients of $\left(\left(\begin{array}{c}n \\ k-i\end{array}\right)-\left(\begin{array}{c}n \\ k-i-1\end{array}\right)\right)$ in the sums for $\operatorname{dim}\left(\operatorname{ker} \delta^{(q)}\right)$ and $\operatorname{dim}\left(\operatorname{im} \delta^{(p)}\right)$.

Since by hypotheses, $B(p) \cap B(q) \neq \varnothing$, then it is easy to see that the only indices $i$ which contribute to the sum satisfy (5.73).

Condition (5.73) can be expressed in words as saying that in the binary expansions of $p, q$ and $i$, some 0 of $i$ corresponds to a 1 of $p$, and some 1 of $i$ corresponds to a 1 of $q$. Of course, if $p=q=2^{t}$ then no such $i$ exists, 
so that the sum in (5.72) is empty, $d(p, q ; k)=0$, and $H_{k}^{p, q}$ is trivial (as we already know by Theorem 1 ).

\section{Applications}

In this section are describe several applications of the preceding ideas, which in fact provided some of our initial motivation for investigating cohomological aspects of hypergraphs.

To begin with, given a $k$-graph $G=(V, E)$ we define the multiplicative edge function $\mu=\mu_{G}: V^{k} \rightarrow\{1,-1\}$ by setting

$$
\mu\left(x_{1}, \ldots, x_{k}\right)=\left\{\begin{aligned}
-1 & \text { if }\left\{x_{1}, \ldots, x_{k}\right\} \in E, \\
1 & \text { otherwise. }
\end{aligned}\right.
$$

With $|V|=n$, we define the deviation of $G$, denoted by $\operatorname{dev} G$, by

$$
\operatorname{dev} G:=\frac{1}{n^{2 k}} \sum_{\substack{v_{i}(0), v_{i}(1) \in V \\ 1 \leq i \leq k}} \prod_{\substack{\varepsilon_{j} \in\{0,1\} \\ 1 \leq j \leq k}} \mu\left(v_{1}\left(\varepsilon_{1}\right), \ldots, v_{k}\left(\varepsilon_{k}\right)\right) .
$$

It turns out that $\operatorname{dev} G$ is a fundamental invariant of a $k$-graph $G$, and gives in many ways a quantitative measure of how much $G$ behaves like a "random" $k$-graph $G_{1 / 2}$ on $V$ (e.g., one in which each $k$-set $X \in\left(\begin{array}{l}V \\ k\end{array}\right)$ is selected independently with probability $1 / 2$ to be an edge of $G_{1 / 2}$ ). In particular, $0 \leq \operatorname{dev} G \leq 1$ always holds, and the closer $\operatorname{dev} G$ is to 0 , the more like a random $k$-graph $G$ is. Families of $k$-graphs $G^{(k)}(n)$ for which $\operatorname{dev} G^{(k)}(n) \rightarrow 0$ as $n \rightarrow \infty$ are called quasi-random. (For a fuller discussion of these ideas, the reader can consult [CGW89, CG90, CG91].)

In [CG91], it was important to characterize those $k$-graphs $G^{(k)}$ with the largest possible deviation, i.e., satisfying

$$
\operatorname{dev} G^{(k)}=1 \text {. }
$$

The following result gives such a characterization.

\section{Theorem 6.}

$$
\operatorname{dev} G^{(k)}=1
$$

if and only if

$$
G^{(k)}=\sum_{i=1}^{k} \delta^{(i)} K^{(k-i)}
$$

for some choice of $(k-i)$-graphs $K^{(k-i)}, 1 \leq i \leq k$.

For a proof, see [CG91].

One property a quasi-random family of $k$-graphs $G^{(k)}(n)=\left(V_{n}, E_{n}\right), n \rightarrow$ $\infty$, satisfies is the following. For any fixed $k$-graph $H^{(k)}(m)=(V, E)$, the number $\#\left\{H^{(k)}(m)<G^{(k)}(n)\right\}$ of maps $\lambda: V \rightarrow V_{n}$ such that $X \in E \Leftrightarrow$ $\lambda(X) \in E_{n}, X \in\left(\begin{array}{l}V \\ k\end{array}\right)$, satisfies

$$
\#\left\{H^{(k)}(m)<G^{(k)}(n)\right\}=(1+o(1)) n^{m} / 2^{\left(\begin{array}{c}
m \\
k
\end{array}\right)}, \quad n \rightarrow \infty .
$$

In other words, all $k$-graphs on a fixed number $m$ of vertices occur (asymptotically) equally often as induced subgraphs of $G^{(k)}(n)$ as $n \rightarrow \infty$. In fact, it 
is shown in [CG91] that if this holds for all $H^{(k)}(2 k)$ on $2 k$ vertices, then it holds for all $H^{(k)}(m)$ for any fixed $m$. Furthermore, the value $2 k$ is critical, in the sense that there exist non-quasi-random families $G^{(k)}(n)$ for which

$$
\#\left\{H^{(k)}(s)<G^{(k)}(n)\right\}=(1+o(1)) n^{s} / 2^{\left(\begin{array}{l}
s \\
k
\end{array}\right)}, \quad n \rightarrow \infty,
$$

for all $s \leq 2 k-1$.

One way of constructing such families for the case $s=2 k-1$, when $k \neq 2^{a}$ for any $a$, is the following.

For $1 \leq t \leq k-1$, choose a "random" $t$-graph $G_{1 / 2}^{(t)}$ on a set $V_{n}$ of size $n$, i.e., each $t$-set $X \in\left(\begin{array}{c}V_{n} \\ t\end{array}\right)$ is designated as an edge of $G_{1 / 2}^{(t)}$ independently with probability $1 / 2$. Define

$$
\hat{G}^{(k)}(n)=\sum_{t=1}^{k-1} \delta^{(t)} G_{1 / 2}^{(k-t)}(n) .
$$

Theorem 7 [CG91]. For almost all choices of $G_{1 / 2}^{(t)}(n), \hat{G}^{(k)}(n)$ satisfies (6.76).

In the case that $k=2^{t}$, a slight extension of this construction gives the required family (see [CG91] for details).

\section{CONCLUding REMARKS}

It would be natural to investigate the corresponding results for more general coefficient groups, e.g., $\mathbb{Z} / p$ or $\mathbb{Z}$, as opposed to $\mathbb{Z} / 2$, which is the simplest choice (and the one for which we had natural applications). A good beginning in this direction has very recently been taken by Dale Darrow, to whom we also wish to thank for a careful reading of an earlier draft of this paper. It would appear that the continuation of these investigations in the directions of the work of Cameron [C77, C78] and others (who dealt with the case $k=3$ ) looks quite promising.

\section{REFERENCES}

[B89] C. Berge, Hypergraphs, North-Holland, Amsterdam, 1989.

[C77] P. J. Cameron, Cohomological aspects of two-graphs, Math. Z. 157 (1977), 101-119.

[C78] _ Automorphisms and cohomology of switching classes, J. Combin. Theory Ser. (B) 22 (1977), 297-298.

[CW86] Y. Cheng and A. L. Wells, Jr., Switching classes of directed graphs, J. Combin. Theory Ser. (B) 40 (1986), 169-186.

[CG90] F. R. K. Chung and R. L. Graham, Quasi-random hypergraphs, Random Structures and Algorithms 1 (1990), 105-124.

[CG91] _ Quasi-random set systems, J. Amer. Math. Soc. 4 (1991), 151-196.

[CGW89] F. R. K. Chung, R. L. Graham and R. M. Wilson, Quasi-random graphs, Combinatorica 9 (1989), 345-362.

[F90] P. Frankl, Intersection theorems and mod $p$ rank of inclusion matrices, J. Combin. Theory Ser. (A) 54 (1990), 85-94.

[GLL80] R. L. Graham, S. Y. R. Li and W. C. Li, On the structure of t-designs, SIAM J. Algebraic and Discrete Method 1 (1980), 8-14.

[GKP89] R. L. Graham, D. E. Knuth and O. Patasknik, Concrete mathematics, Addison-Wesley, Reading, Mass., 1989. 
[GRS90] R. L. Graham, B. L. Rothschild and J. H. Spencer, Ramsey theory, (2nd ed.), Wiley, New York, 1990.

[GJ73] J. E. Grover and W. B. Jurkat, The module structure of integral designs, J. Combin. Theory Ser. (A) 15 (1973), 75-90.

[H49] S. T. Hu, A cohomology theory with higher coboundary operators. I (Construction of the groups), Nederl. Akad. Wetensch. Proc. 52 (1949), 1144-1150.

[H50] A cohomology theory with higher coboundary operators. II (Verification of the axioms of Eilenberg-Steenrod), Nederl. Akad. Wetensch. Proc. 53 (1950), 70-76.

[H52] _ A cohomology theory with higher coboundary operators. III (The homotopy axiom and the groups for spheres), Nederl. Akad. Wetensch. Proc. 55 (1952), 123-129.

[K72] W. M. Kantor, On incidence matrices of finite projective and affine spaces, Math. Z. 124 (1972), 315-318.

[LR81] N. Linial and B. L. Rothschild, Incidence matrices of subsets - a rank formula, SIAM J. Algebraic and Discrete Methods 2 (1981), 333-340.

[MS75] C. L. Mallows and N. J. A. Sloane, Two-graphs, switching classes and Euler graphs are equal in number, SIAM J. Appl. Math. 28 (1975), 876-880.

[ML83] W. Mielants and $\mathrm{H}$. Leemans, $\mathbb{Z}_{2}$-cohomology of projective spaces of odd order, Combinatorics '81 (Rome, 1981), North-Holland Math. Stud., 78, North-Holland, Amsterdam, 1983, pp. 635-651.

[M84] J. R. Munkres, Elements of algebraic topology, Benjamin/Cummings, Menlo Park, Calif., 1984.

[S76] J. J. Seidel, A survey of two-graphs, Colloquio Internazionale sulle Teorie Combinatorie, vol. 1, Accademia Nazionale dei Lincei, Rome, 1976, pp. 481-511.

[ST81] J. J. Seidel and D. E. Taylor, Two-graphs, a second survey, Algebraic Methods in Graph Theory (L. Lovász and V. T. Soś, eds.), North-Holland, Amsterdam, 1981.

[We84] A. L. Wells, Jr., Even signings, signal switching classes and (-1, 1)-matrices, J. Combin. Theory Ser. (B) 36 (1984), 194-212.

[Wi*] R. M. Wilson, $A$ diagonal form for the incidence matrices of $t$-subsets vs. $k$-subsets, European J. Combin. 11 (1990), 609-615.

[Z81] T. Zaslavsky, Characterization of signed graphs, J. Graph. Theory 5 (1981), 401-406.

Bell Communications Research, Morristown, New Jersey 07960

E-mail address: frkc@bellcore.com

AT\& Bell Laboratories, Murray Hill, New Jersey 07974

E-mail address: rlg@research.att.com 